This paper is published in the open archive of Mid Sweden University

DIVA http://miun.diva-portal.org

with permission of the publisher

Citation for the peer-reviewed published paper:

Hellström L, Gradin P, Carlberg T. A Method for Experimental Investigation of the Wood Chipping Process. ; Nordic Pulp \& Paper Research Journal. $2008 ; 23(3): 339-342$.

URL to article at publishers site:

http://dx.doi.org/10.3183/NPPRJ-2008-23-03-p339-342 


\title{
A method for experimental investigation of the wood chipping process
}

\author{
Lisbeth M. Hellström, Per A. Gradin and Torbjörn Carlberg, Mid Sweden University, Sundsvall, Sweden
}

KEYWORDS: Wood chipping, Digital Speckle Photography, Friction, Fracture processes

SUMMARY: To be able to formulate criteria for determining the onset of the creation of a wood chip; one should be able to study the deformation fields in the vicinity of the edge of the chipping tool. To that end, an experimental setup was developed in which the chipping can be performed under very well defined conditions. In this setup it is possible to control the rate of indentation of the chipping tool and also to measure the force on the tool. The setup even allows the angle of the wood specimen with respect to the cutting plane to be varied in both a horizontal and a vertical plane.

To determine the deformations, a Digital Speckle Photography (DSP) equipment is used, which together with image processing software makes it possible to determine the strain field on the surface of the wood specimen. One observation that can be made in these studies is that prior to the creation of a chip, there is a localization of strains in a thin region of the wood, starting from the edge of the tool and directed parallel to the grain.

Another observation made during the experimental work is that there exist different types of fracture processes, each giving different chip thicknesses.

\section{ADDRESS OF THE AUTHORS:}

Lisbeth M. Hellström (lisbeth.hellstrom@miun.se), Per A. Gradin (per.gradin@miun.se) and Torbjörn Carlberg (torbjorn.carlberg@miun.se): Mid Sweden University, Fibre Science and Communication Network, FSCN, Department of Engineering, Physic and Mathematic, Holmgatan 10, SE-851 70 Sundsvall, Sweden.

Corresponding author: Lisbeth M. Hellström

The total worldwide production of virgin wood pulp amounts to about 180 million tons a year. This includes both chemical and mechanical pulp. Common for both of these types of pulp is that the raw material (the logs) has to be cut into small parts i.e. chips before the fibres can be separated. If it is assumed that $70 \%$ of the weight of chips becomes pulp for example in the chemical pulping process, as some of the material is dissolved during the chemical treatment, then it can be concluded that about 260 million tons of chips are produced annually. From this it follows that the chipping process is an important part of the pulping process.

A demand from the pulp and paper industry is that the chip quality should be as uniform as possible. It seems to be a consensus that the primary quality requirement of the chips is that they should vary as little as possible in their thickness distribution. This view on quality comes from e.g. that in order to produce chemical pulp, the chips have to be impregnated with chemicals, and with a very narrow thickness distribution all chips are uniformly impregnated. If so, the quality of the pulp will also become very uniform.

The important thing is not primarily to produce chips with a specific thickness but that the thickness is as uniform as possible. This means that it is not necessarily so that the chipping tool should be as sharp as possible but rather that the chipping tools retain their characteristics over time. To be able to predict the impact of for example tool wear on the chip thickness, one has to have a detailed understanding of the underlying mechanisms of chip formation. This study is an attempt to device a tool for studying these mechanisms.

Investigations carried out previously have concentrated on e.g. measuring the forces on the chipping tool (at low velocities) (Uhmeier 1995; Buchanan, Duchnicki 1963); measuring chip thicknesses when varying different process parameters etc (Buchanan, Duchnicki 1963; Kivimaa, Murto 1949; Hartler 1962a-c). Very little seems to have been done with respect to the study of local parameters. Interesting in this context are the local deformation fields i.e. the strain distributions in a vicinity of the chipping tool i.e. the knife edge. One way to accomplish this is by using a Digital Speckle Photography (DSP) equipment. Such a system, used for the deformation analysis, is based on analyses of the images of the undeformed and deformed wood specimen from which chips are cut. By taking photographs of the surface, and using image processing of a specific geometry both in an unloaded and loaded state, it is possible to estimate the displacements in a pre - defined region. By numerical differentiation of the displacement field, the strain field can be determined. A necessary condition for application of the technique is, however, that the surface has a clearly identifiable structure. DSP has found large applicability in a number of interesting fields. The method has been used among other things e.g. for estimating hygro - expansion of paper (Lif et al. 1995), visco - plastic properties of metals (Kajberg, Wikman 2007) and elastic properties of wood fibre walls (Bergander, Salmén 2000). Thuvander et al. (2000) used it to study crack tip strain field in wood at the scale of annual growth rings, Jernkvist and Thuvander (2001) studied stiffness variation across annual growth rings in Picea Abies. Ljungdahl et al. (2006) studied transverse anisotropy of compressive failure in European oak and Dumail et al. (2000) analyzed rolling shear of spruce wood. Using this technique to study local parameters in connection with the wood chipping process is (to our knowledge) a novel approach.

By using DSP it is possible to evaluate quantitatively the in-plane displacement and strain fields. By eliminating the rigid body movement of the specimen, the local deformations on the specimen surface can be determined. Of interest are the local deformations (strains), up to the 
instant when a chip is formed. Depending on the size of the selected test area, smaller cracks will be captured and visualized as local strain concentrations.

The cutting rate in this investigation is low in comparison to normal industrial cutting. The cutting speed has a significant effect on pin chip production and even on the production of over thick chips (Hartler 1986; Smith, Javid 1999). Low chipping speed almost certainly results in a high sliding frictional component of the work of chipping (Buchanan, Duchnicki 1963). There are other parameters which affect the material behaviour such as temperature, moisture content etc., which have not been considered in this investigation.

The results from the DSP measurements will make it possible to compare the results obtained by using some numerical scheme e.g. the Finite Element Method (FEM) together with some assumed constitutive model for the wood material.

\section{Experimental Setup}

To perform the chipping in a well-defined way, a special fixture was designed. A photo of the experimental setup is shown in Fig 1. The ARAMIS measuring system (GOM mbH 20040820) was used for the deformation analysis of the specimen surface for determining the fracture behaviour including the crack development. A hydraulic testing machine (MTS) was used to load the chipping tool. To fix the wood sample, a specimen holder was used. The specimen holder admitted a variation of the cutting angles in both a horizontal and a vertical plane. The whole chipping device is shown in Fig 2.

The loading of the chipping tool is accomplished by mounting the whole fixture in a servo hydraulic testing machine (MTS) and a $50 \mathrm{kN}$ load cell was used to measure the applied force. Displacement-controlled testing was performed with a crosshead speed of 1.0 $\mathrm{mm} / \mathrm{s}$. In this way, the cutting rate can be controlled accurately and the force on the chipping tool can be measured. In Fig 1 is also shown the camera belonging to the DSP equipment. As mentioned previously, the DSP method requires an identifiable surface structure. To that end, the surface of the wood specimen was first painted white and then sprayed with black colour to get a random pattern of black dots. The technique relies on that the

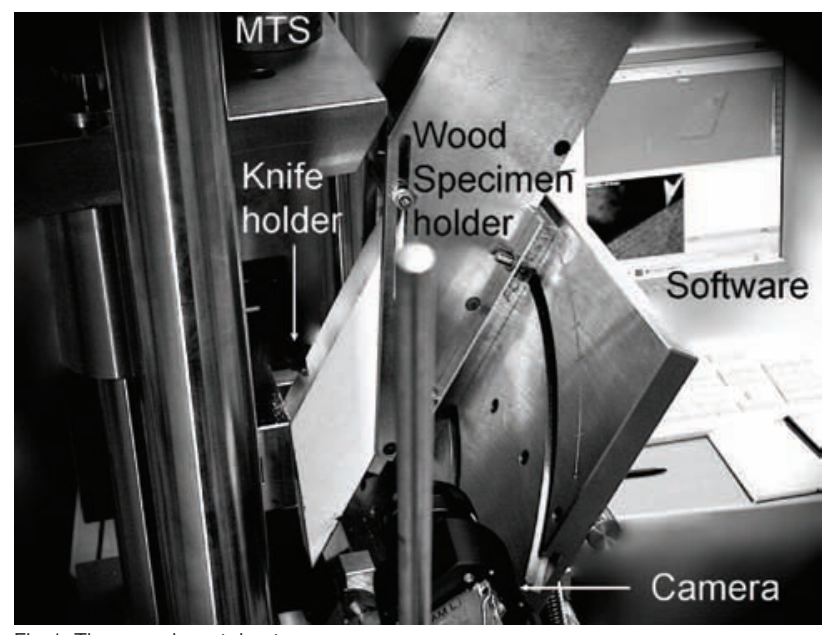

Fig 1. The experimental setup. motion of this pattern can be detected between frames; therefore the quality of the pattern is crucial.

The charge-coupled-device (CCD) camera focused on the part of the specimen closest to the knife-edge and the software was programmed to take 12 photographs per second for a 2D analysis. The material used in the chipping test was Norway spruce (Picea abies) with a moisture content corresponding to that of green wood.

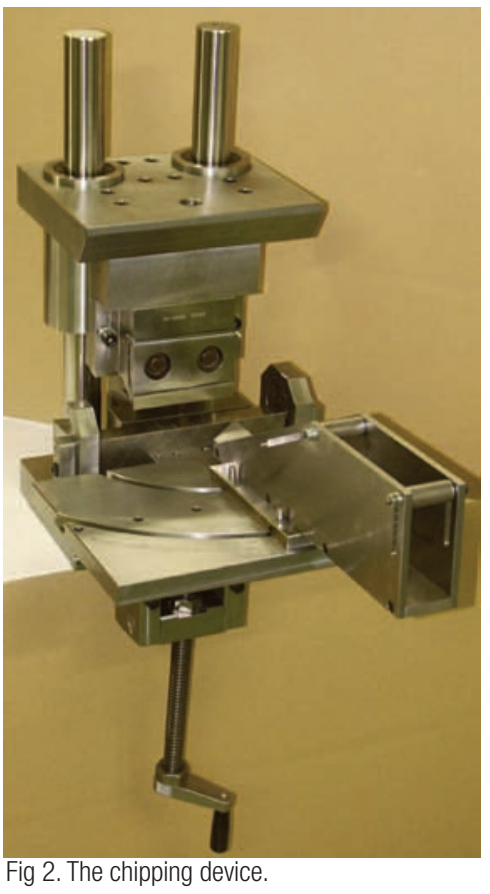
For the test, a specimen with cross section dimensions of $35 \times 82 \mathrm{~mm}^{2}$ was used.

The following cutting angles were chosen: sharpness angle $\beta=34^{\circ}$, clearance angle $\alpha=3^{\circ}$, spout angle $\varepsilon=$ $30^{\circ}$ (Fig 3).

As soon as at least two images of the test surface are captured, the relative displacement between them can be calculated. The software included in the equipment calculates the displacement field and after that, the strain field is obtained by numerical differentiation of the displacement field. For best results, the sample has to be perpendicular to the camera.

\section{Some Results}

An example of the output from a DSP study can be seen in Fig 4, where the normal strain in the radial (R) direction is shown.

In Fig 4, the read colour indicates a thin region with high shear strains just prior to chip formation. In Fig 5,

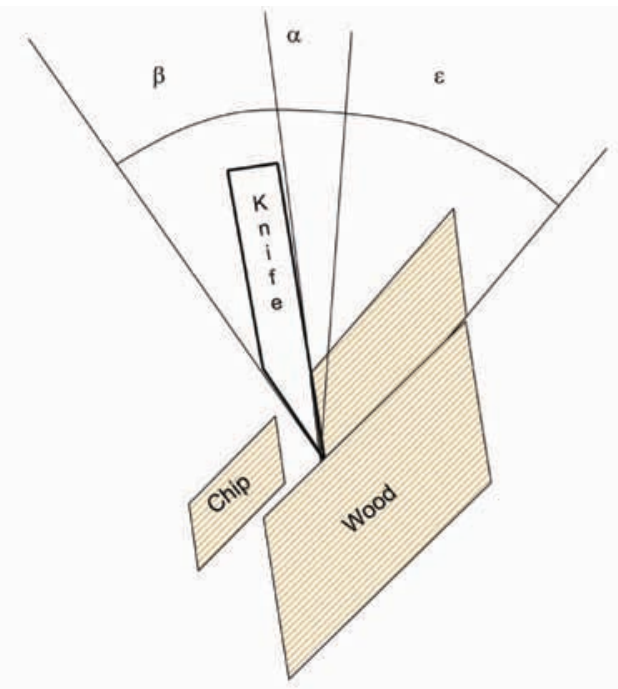

Fig 3. The cutting angles $\alpha, \beta$, and $\varepsilon$. 


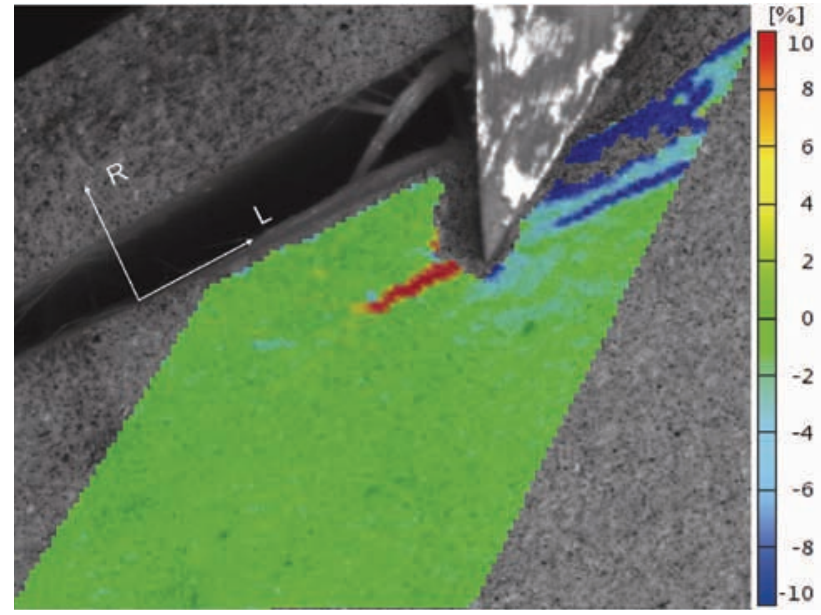

Fig 4. Normal strain distribution in the R-direction (\%).

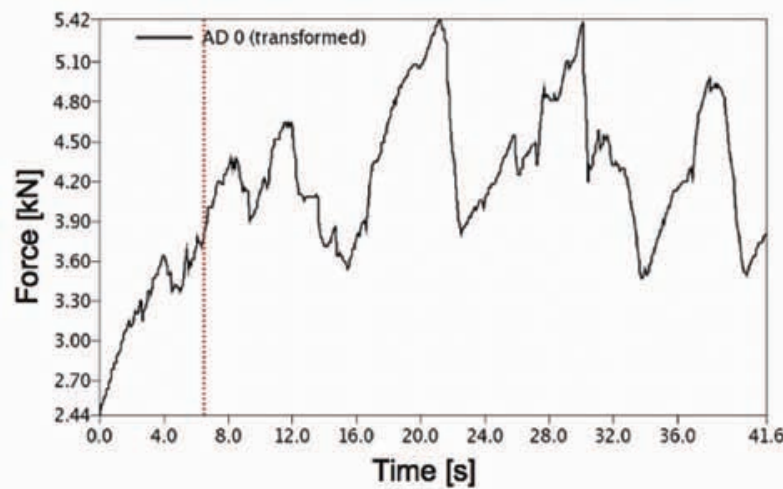

Fig 5.Force vs. time.

the force vs. time (in essence the knife edge position, since the cutting rate is constant) is shown. The red, dotted vertical line refers to the instant for which Fig 4 is relevant. It can be observed that the force curve is composed of large amplitude variations on which smaller variations are superimposed. The large variations correspond to chip formation while the smaller ones correspond to the initiation of smaller sub - critical cracks.

Due to limitations of the camera in the DSP system, the cutting rate had to be kept as low as $1 \mathrm{~mm} / \mathrm{s}$ which is far below the rate used in the chipping process. However, even though it is well known that wood in general shows a rate dependency, it is believed that studies of this kind will shed some light over the basic mechanisms involved in the creating of a wood chip.

During the experiments mainly three different types of chip formation processes have been identified i.e. an opening-modus (Fig 6), a forward shear modus (Fig 7) and a mode according to Fig 8 which in lack of a better term might be referred to as a remote opening mode.

Which of the processes that will be the most frequent one, is largely dependent on the friction between the wood material and the chipping tool. Figs 6,7 and 8 show the influence of friction ranging from low friction in Fig 6 to high friction in Fig 8. To get a chip thickness distribution as narrow as possible, a god start would be to ascertain that only one fracture process is active and this means that the friction between the surface of the chipping tool and the wood should be as low as possible.

Another interesting observation made, is that before a chip or a small sub critical crack has formed, the force vs.

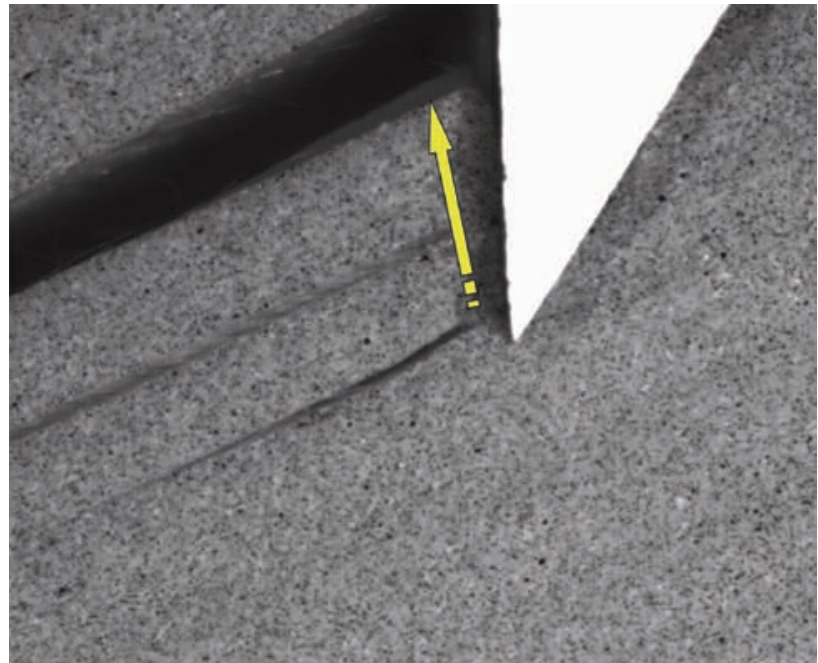

Fig 6. Opening mode.

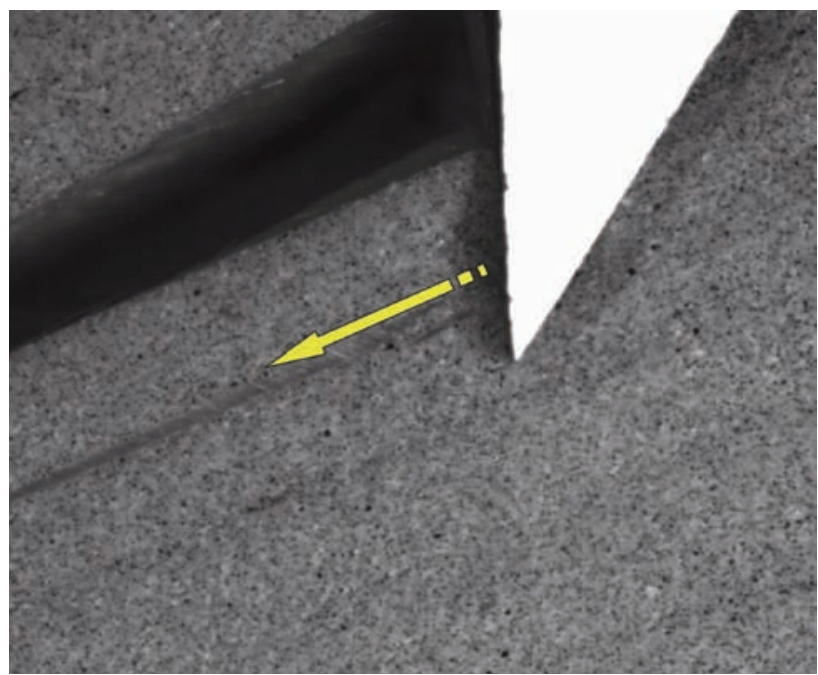

Fig 7. Forward shear mode.

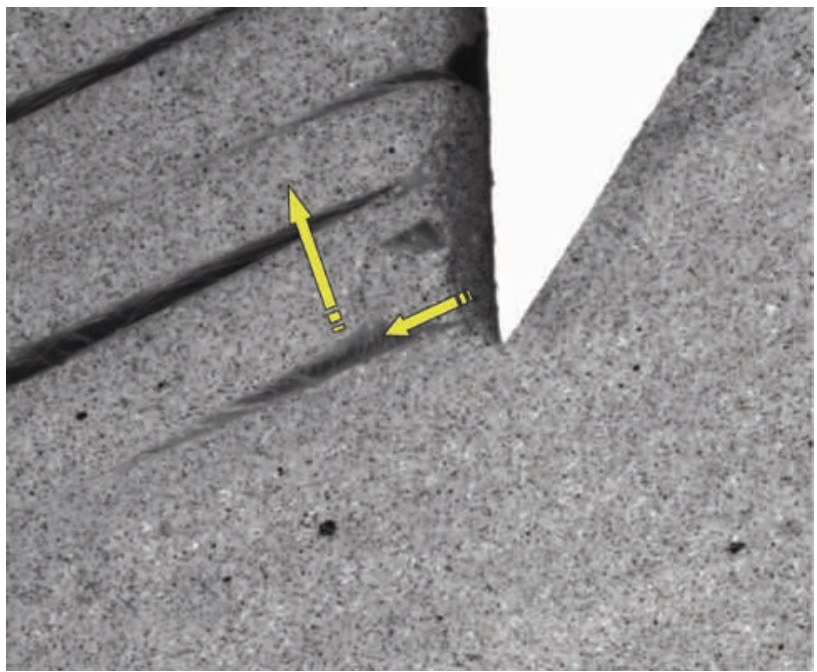

Fig 8. Remote opening mode.

time relation is always almost linear despite the fact that the material behaviour of wood is highly non - linear. Considering a hypothetical situation with a homogeneous semi - infinite structure with no intrinsic length scale, it can be shown that regardless the material behaviour, the load vs. penetration depth is always a linear relation (self similarity) provided that the chipping tool has straight edges. In wood, the assumption regarding the intrinsic length scale is obviously not true since one length scale is 


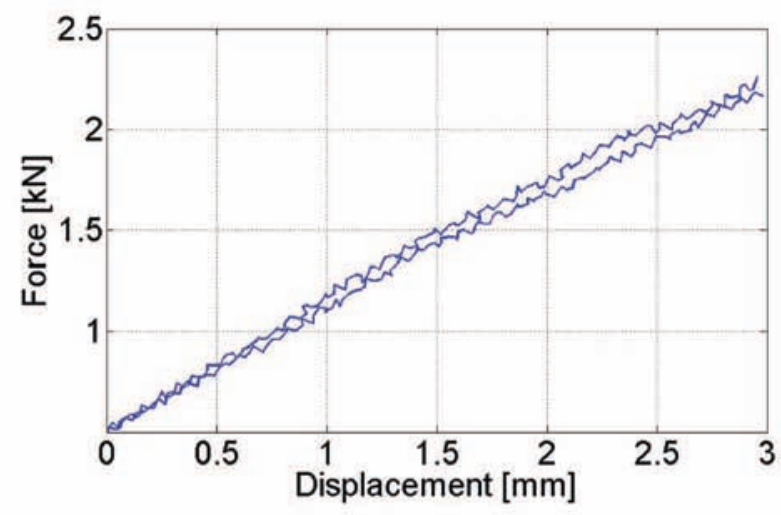

Fig 9. Force vs. displacement for $d=10 \mathrm{~mm}$.

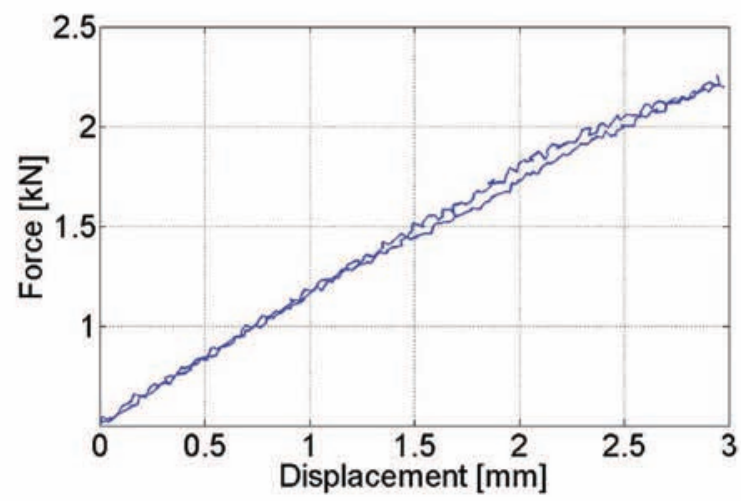

Fig 10. Force vs. displacement for $\mathrm{d}=20 \mathrm{~mm}$.

determined by the annual growth ring structure. Also, the assumption regarding the semi - infinite nature of the problem does not hold in a real situation. In Figs 9 and 10 is shown the force vs. displacement (penetration depth) for two different values of the distance, $d$, from the cutting plane to the free end. In each figure is shown the result from two tests.

It can be observed that even when the assumptions regarding homogeneity and semi - infinity are violated, the cutting process is approximately self - similar.

\section{Discussion and Conclusions}

It can be concluded that the experimental procedure described in this article is a versatile tool when it comes to studying the chipping process and in particular the local strain fields in the vicinity of the cutting edge of the chipping tool. Based on the results from this preliminary investigation it can be concluded that just prior to the formation of a chip, there is a concentration of strains in a narrow zone in a thin region starting from the edge of the tool and directed parallel to the grain.

Another conclusion is that the friction between the wood and the chipping tool is probably one crucial factor for the chip formation process. Finally it is observed that the indentation process is approximately self - similar.

Apart from e.g. the friction, the process of chip formation is also greatly influenced by the mechanical properties of the wood and it is obvious that it is impossible to get a smaller variation in the chip thickness than is dictated by the inherent variations in the wood material.

Another benefit by having access to the strain distributions obtained by the experimental procedure described here is that Finite Element (FE) calculations based on different assumptions regarding the mechanical behaviour of wood can be checked against the measured strains so that the quality of the assumptions can be judged.

Since the DSP method gives the strains only, one has to rely on e.g. the FE - method to obtain the stress distributions, which will be needed to judge for example the influence of opening stresses in relation to shear stresses on the chip formation process, which is the topic of a forthcoming study.

The present method is aimed for studying deformation processes that are active in wood during chip formation. As mentioned before the cutting speed in this investigation is low in comparison to what is normally used. In a forthcoming study, chip formation processes will be studied at commonly used cutting speeds $(20-40 \mathrm{~m} / \mathrm{s})$ by utilising a laboratory disc chipper.

\section{Acknowledgements}

The Swedish KK-Foundation is acknowledged for financial support. Iggesund tools AB, Holmen Paper, and Staffan Nyström at the Mid Sweden University are greatly acknowledged for their support with the development of the experimental equipment.

\section{Literature}

Uhmeier, A. (1995): Some fundamental aspects of wood chipping, Tappi J. 78: 10, 79-86

Buchanan, J.G. and Duchnicki, T.S. (1963): Some Experiments in Low-Speed Chipping, Pulp Paper Mag. Can. May 1963, T235-T245.

Kivimaa, E. and Murto, J.0. (1949): Investigations on factors affecting chipping of pulpwood, Statens Tekniska Forskningsanstalt, Finland, Publ. 9.

Hartler, N. (1962a): The Effect of Spout Angle as Studied in an Experimental Chipper, Svensk Papperstidning, 65: 9, 351-362.

Hartler, N. (1962b): The effect of Sharpness Angle as Studied in an Experimental Chipper, Svensk Papperstidning, 65: 10, 397-402.

Hartler, N. (1962c): Studies on the Suction Feeding of Chippers, Svensk Papperstidning, 65: 12, 475-487.

Twaddle, A. (1997): The influence of species, chip length, and ring orientation on chip thickness, Tappi J. 80: 6, 123-131.

Lif, J.0., Fellers, C., Soremark, C. Sjodahl, M. (1995): Characterizing the inplane hygroexpansivity of paper by electronic speckle photography, J. Pulp Paper Sci. Vol. 21 No. 9: J302-J309.

Kajberg, J. and Wikman, B. (2007): Viscoplastic parameter estimation by high strain-rate experiments and inverse modelling - Speckle measurements and highspeed photography, Int. J. of Solids and Structures, 44: 145-164.

Bergander, A., Salmen, L. (2000): Transverse elastic modulus of native wood fibre wall, J. Pulp Paper Sci. Vol. 26 No. 6: 234-238.

Thuvander, F., Sjödahl, M. and Berglund, L.A. (2000): Measurement of crack tip strain field in wood at scale of growth rings, J. Mater. Sci. 35: 6267-6275.

Jernkvist, L.0. and Thuvander, F. (2001): Experimental Determination of Stiffness Variation Across Growth Rings in Picea abies, Holzforschung, 55: 309-317.

Ljungdahl, J., Berglund, L.A. and Burman, M. (2006): Transverse anisotropy of compressive failure in European oak - a digital speckle photography study, Holzforschung, 60: 190-195.

Dumail, J.-F., Olofsson, K. and Salmén, L. (2000): An Analysis of Rolling Shear of Spruce Wood by the losipescu Method, Holzforschung, 54: 420-426.

Hartler, N. (1986): Chipper design and operation for optimum chip quality, Tappi J. 69: 10, 62-66.

Smith, D. and Javid, S.J. (1999): Improve Chipper design Yields Better Chips for Chemical Pulping, Pulp and Paper, July 1999, 54-56.

GOM mbH (2004-08-20): ARAMIS user manual, en Rev A; Germany. 